\title{
Generating the Schrödinger cat state in a nanomechanical resonator coupled to a charge qubit
}

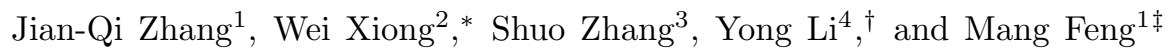 \\ ${ }^{1}$ State Key Laboratory of Magnetic Resonance and Atomic and Molecular Physics, \\ Wuhan Institute of Physics and Mathematics, Chinese Academy of Sciences, Wuhan 430071, \\ China ${ }^{2}$ Department of Physics and State of Key Laboratory of Surface Physics, Fudan University, Shanghai 200433, China \\ ${ }^{3}$ College of Science, National University of Defense Technology, Changsha 410073, China \\ ${ }^{4}$ Beijing Computational Science Research Center, Beijing 100084, China
}

\begin{abstract}
We propose a scheme for generating the Schrödinger cat state based on geometric operations by a nanomechanical resonator coupled to a superconducting charge qubit. The charge qubit, driven by two strong classical fields, interacts with a high-frequency phonon mode of the nanomechanical resonator. During the operation, the charge qubit undergoes no real transitions, while the phonon mode of the nanomechanical resonator is displaced along different paths in the phase space, dependent on the states of the charge qubit. This generates the entangled cat state between the NAMR and charge qubit, and the cat state for the superposition of NAMR can be achieved after some operations applied on this entangled cat state. The robustness of the scheme is justified by considering noise from environment, and the feasibility of the scheme is discussed.
\end{abstract}

PACS numbers:

\section{INTRODUCTION}

Recently, nanomechanical resonators (NAMRs) have attracted considerable attention both theoretically and experimentally, due to the fact that NAMRs own both classical and quantum properties [1, 2], which provides unique opportunity for studying quantum properties in macroscopic objects.

Generating large quantum superposition of the macroscopic objects is an essential task in the field of the macroscopic quantum mechanics [3 6], which provides a good platform to understand the mechanism of decoherence in macroscopic objects [7, 8], to check the scope of application of quantum theory [9] and to observe the transition between quantum and classical physics [10]. To this end, the large quantum superposition, like the Schrödinger cat state (simply called 'cat state' in the following), has been realized in various systems, such as trapped ions [11], photons [12], superconducting qubits [13], macroscopic current [14], and NAMR [15]. However, those schemes are based on the dynamical evolution which is sensitive to quantum fluctuations and definitely unsuitable for preparing macroscopic quantum states. Therefore, it is desirable to find a robust way to creating the cat state against the quantum fluctuation.

On the other hand, the geometric phase is only determined by the path of the state evolution, rather than the initial state distribution or any details of the path [1619]. In this way, operations and processes based on the geometric phases are robust to the fluctuation and some other imperfections in the evolution [11 13, 15, 20]. So

\footnotetext{
*Electronic address: boing777@qq.com

† Electronic address: liyong@csrc.ac.cn

$\ddagger$ Electronic address: mangfeng@wipm.ac.cn
}

far there are two kinds of geometric phases. The one taking no dynamical phase is called conventional geometric phase [21], where removing the dynamical phase from the evolution is essential to the related operations. As a result, although the geometric phase is intrinsically resistant to parameter fluctuation, the additional operations for eliminating the dynamical phases usually bring in unexpected errors. In contrast, the so-called unconventional geometric phase [16] remains a non-zero dynamical phase proportional to the geometric phase by a constant independent of the parameters. As a result, despite involvement of the dynamical phase, all geometric advantages are still possessed in the processing with unconventional geometric phase.

In this work, we demonstrate the possibility to generate the cat state by displacement operators from the unconventional geometric phase gate (defined later) in the hybrid system consisting of a charge qubit [22] and a NAMR. Although we have realized that the unconventional geometric phase has been achieved in the system of trapped ions [23], which is analogous to the model under our consideration, the method working there is not applicable to the NAMR due to much bigger mass of the NAMR making the coupling to the charge qubit negligible. However, it is worth pointing out that the unconventional geometric phase is not robust against the qubit decay, i.e., the charge noise. To overcome this problem, we consider a transmon-type charge qubit in our work, which is insensitive to the charge noise due to a large ratio of the Josephson energy to the charging energy [24]. The decay time of this kind of charge qubit can reach 0.1 ms experimentally [25].

Different from the previous schemes involving the unconventional geometric phase [16, 18, 19, 23, 26, 27] for quantum computation, our scheme focuses on generating the cat states based on the displacement operators in a geometric fashion, which guarantees the robustness 
of the cat states of the NAMR. Moreover, in comparison with a recent proposal [4] in which the cat state is created in an optomechanical cavity, the cat state generated in our hybrid system composed of a charge qubit and a NAMR can be more 'macroscopic'. In addition, there is a controllable coupling between the NAMR and the charge qubit, which can be adjusted by the bias voltage on the NAMR. Therefore, the NAMR and the charge qubit can be decoupled after the cat state is generated. As a practical aspect, we suppose that the NAMR owns a very high frequency, which ensures the NAMR remaining in the ground state in the work temperature of the charge qubit. Assisted by the operations based on the unconventional geometric phase with a transmon-type charge qubit, our scheme is robust against the fluctuations from the external light field within the coherence time, and very promising for achievement using currently available techniques.

The paper is organized as follows. In the next section, we introduce the theoretical model and derive the effective Hamiltonian. In Sec. III, we review the implementation of an unconventional geometric phase gate. Generation of the cat states based on the unconventional geometric phase is discussed in Sec. IV] and we justify the robustness of our scheme in Sec. V] A brief conclusion is given in Sec. VI]

\section{THEORETICAL MODEL AND THE EFFECTIVE HAMILTONIAN}

As shown in Figure 1, a metallic NAMR which takes mass $m$, frequency $\omega_{m}$ and length $L$ is coupled to a superconducting charge qubit with effective Josephson energy $E_{J}$ and junction capacitance $C_{J}$ by the capacitor $C(x)$ after a static voltage $V_{x}$ is applied. To suppress the charge noise of the charge qubit, we suppose that the qubit is well isolated from the rest circuitry by an additional capacitance $C_{i n}$, forming a transmon-type charge qubit 24]. The capacitor $C(x)$ depends on the displacement $x$ of the NAMR around its equilibrium position. The charge qubit is not only controlled by a DC voltage $V_{0}$, but also driven by two AC voltages $V_{g}(t)=V \cos \omega_{l} t$ and $V_{g}^{\prime}(t)=V^{\prime} \cos \omega_{l}^{\prime} t$ via the gate capacitor $C_{g}$. If the charge qubit works in a near optimal point, which means $\left[C_{g} V_{0}+\left.C(x)\right|_{x=0} V_{x}\right] / 2 e \approx 0.5$, and the charge energy $E_{c}$ is much less than the effective Josephson energy $E_{J}$ [24, 30], the charge noise can be effectively suppressed, and the transition frequency between the first excited and ground states is $\omega_{0} \approx \sqrt{8 E_{J} E_{c}} / \hbar[28]$. By neglecting the higher-order terms regarding the multi-photon excitations, the Hamiltonian describing our model in the rotating-wave approximation is given by (in units of $\hbar=1)$ 28, 29]

$$
\begin{aligned}
\hat{H} & =\frac{1}{2} \omega_{0} \sigma_{z}+\omega_{m} a^{\dagger} a+g\left(a \sigma^{+}+a^{\dagger} \sigma^{-}\right) \\
& +\left[\left(\frac{\Omega}{2} e^{-i \omega_{l} t}+\frac{\Omega^{\prime}}{2} e^{-i \omega_{l}^{\prime} t}\right) \sigma^{+}+H . c .\right],
\end{aligned}
$$
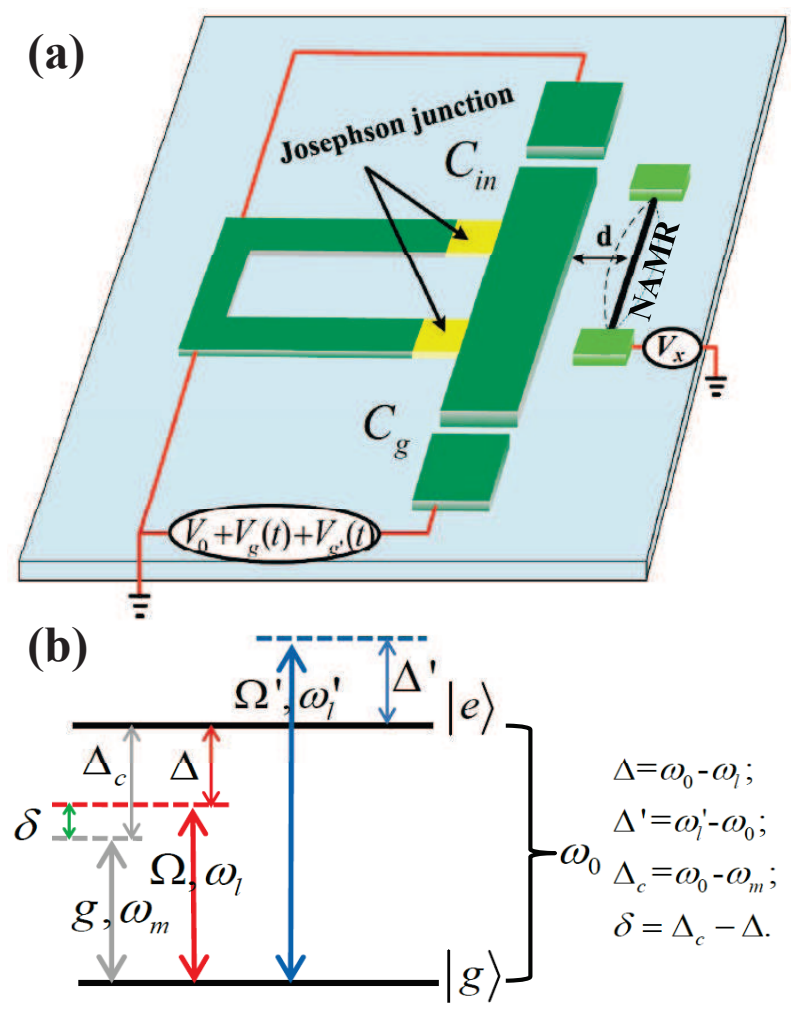

FIG. 1: (a) Schematic diagram of a NAMR (the black thick line on the right-hand side) coupled to a transmon-type charge qubit (on the left-hand side) [24, 28, 29], which could be controlled by an external magnetic field. Two black dashed curves around the NAMR present the NAMR in oscillation. Moreover, there is a bias voltage $V_{x}$ applied on our model, which can be used to control the coupling between the NAMR and the charge qubit. (b) The corresponding energy levels and transitions in our scheme, where the parameters are defined in the text.

where $\sigma_{z}=|e\rangle\langle e|-| g\rangle\left\langle g\left|, \sigma^{+}=\right| e\right\rangle\langle g|$, and $\sigma^{-}=|g\rangle\langle e|$ with the excited and ground states $|e\rangle$ and $|g\rangle$ in the charge qubit, respectively. The operator $a\left(a^{\dagger}\right)$, which is the annihilation (creation) operator of the NAMR, can be written as

$$
\begin{aligned}
a & =\sqrt{\frac{m \omega_{m}}{2}}\left(x+\frac{i}{m \omega_{m}} p\right), \\
a^{\dagger} & =\sqrt{\frac{m \omega_{m}}{2}}\left(x-\frac{i}{m \omega_{m}} p\right),
\end{aligned}
$$

with $p$ being the momentum operator of the NAMR. In Eq. (1), the first two terms describe the free Hamiltonians of the charge qubit and the NAMR, respectively. The third term shows the interaction relevant to the bias voltage $V_{x}$, i.e., a capacitive coupling between the NAMR and the charge qubit, with the strength

$$
g=\frac{4 E_{c} N_{x} X_{0}}{d}
$$

governed by the charging energy $E_{c}$, the Copper pair number $N_{x}=C(0) V_{x}$, the zero-point motion amplitude 
of the NAMR $X_{0}=1 / \sqrt{2 m \omega_{m}}$, and the distance between the charge qubit and the NAMR $d$. The last two terms describe the qubit driven by two classical fields with the frequencies $\omega_{l}$ and $\omega_{l}^{\prime}$, and the Rabi frequencies $\Omega$ and $\Omega^{\prime}$, respectively.

In the interaction picture, the Hamiltonian can be rewritten as

$$
\hat{H}_{I}=\left(g a e^{i \Delta_{c} t}+\frac{\Omega}{2} e^{i \Delta t}+\frac{\Omega^{\prime}}{2} e^{-i \Delta^{\prime} t}\right) \sigma^{+}+H . c .,
$$

with the detunings for the phonon mode of the NAMR and the classical fields from the charge qubit, respectively, i.e., $\Delta_{c}=\omega_{0}-\omega_{m}, \Delta=\omega_{0}-\omega_{l}$, and $\Delta^{\prime}=$ $-\left(\omega_{0}-\omega_{l}^{\prime}\right)$ [see Figure 1(b)].

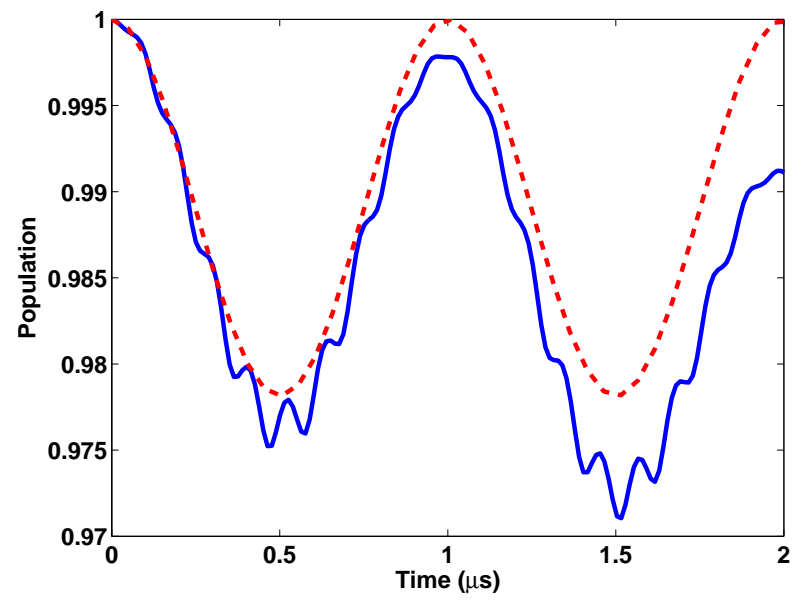

FIG. 2: (Color online) Time evolution of the population of the state $(|g\rangle+|e\rangle) / \sqrt{2}$, where the states for the charge qubit and the nanomechanical mode are initially in the superposition state $(|g\rangle+|e\rangle) / \sqrt{2}$ and in a vacuum state $|0\rangle$, respectively. Other parameters are $g=2 \pi \times 3 \mathrm{MHz}, \Omega=\Omega^{\prime}=2 \pi \times 30$ $\mathrm{MHz}, \Delta_{c}=2 \pi \times 302 \mathrm{MHz}$, and $\Delta=\Delta^{\prime}=2 \pi \times 300 \mathrm{MHz}$. The red dashed and blue solid curves are plotted for comparison, by the Hamiltonians in Eq. (5) and Eq. (4), respectively.

To generate the entanglement state for the NAMR cat state based on the displacement operators, we derive below an effective Hamiltonian by employing the method proposed in Refs. [18, 27, 31, 32] under the following conditions: (1) $|\Omega|=\left|\Omega^{\prime}\right| ;(2) \Delta=\Delta^{\prime}>0 ;$ (3) $\Delta_{c}>\Delta \gg\left\{|g|,|\Omega|,\left|\Omega^{\prime}\right|\right\} ;(4)|\Omega| \gg|g|$. The first condition together with the second condition can completely cancel the Stark shifts caused by the two driving fields and other related terms. Under the large detuning condition (3), the charge qubit, initially in the ground (excited) state, only virtually exchanges phonons with the field of the NAMR if the operation time is fully within the decoherence time. Moreover, the last condition ensures that the terms proportional to $|g|^{2}$ are negligible. Therefore, we obtain the following effective Hamiltonian

$$
\hat{H}_{e f f}=\left(\lambda a e^{i \delta t}+\lambda^{*} a^{\dagger} e^{-i \delta t}\right) \sigma_{z},
$$

with $\delta=\Delta_{c}-\Delta \ll \Delta$ and $\lambda=\frac{\Omega g}{4}\left(\frac{1}{\Delta}+\frac{1}{\Delta_{c}}\right)$. As demonstrated in Figure 2 the effective Hamiltonian (5) presents the main feature of the interaction Hamiltonian (4), particularly within the decoherence times of the transmon-type charge qubit and the NAMR (i.e., $t<50$ $\mu \mathrm{s}[15,25,28,33])$.

\section{THE TOTAL DISPLACEMENT OPERATOR AND THE UNCONVENTIONAL GEOMETRIC PHASE}

We present below how to achieve the total displacement operator and generate the unconventional geometric phase in the NAMR-qubit system using the effective Hamiltonian (5). As a starting point, we describe the derivation of the unconventional geometric phase based on the displacement operator along an arbitrary path in the phase space [16, 17, 23, 26, 27]. For a displacement operator of the bosonic field,

$$
D(\alpha)=e^{\alpha a^{\dagger}-\alpha^{*} a},
$$

where $\alpha$ is the time-dependent displacement parameter for the creation operator $a^{\dagger}$ of the bosonic field (i.e., the phonon mode of the NAMR in this scheme), respectively. After experiencing a path which is divided in the phase space into $N$ short straight sections $\Delta \alpha_{m}$ ( $m=1,2,3, \cdots)$, the total displacement operation is given by [16, 17],

$$
\begin{aligned}
D_{t} & =D\left(\Delta \alpha_{N}\right) \ldots D\left(\Delta \alpha_{1}\right) \\
& =\exp \left(i \operatorname{Im}\left\{\sum_{m=2}^{N} \Delta \alpha_{m} \sum_{k=1}^{m-1} \Delta \alpha_{k}^{*}\right\}\right) D\left(\sum_{m=1}^{N} \Delta \alpha_{m}\right),
\end{aligned}
$$

where the Baker-Campbell-Hausdorff formula has been employed. For $N \longrightarrow \infty$, the total displacement operator is reduced to

$$
D_{t}=e^{i \Theta} D\left(\int_{\gamma} d \alpha\right),
$$

with the total unconventional geometric phase

$$
\Theta=\operatorname{Im}\left\{\int_{\gamma} \alpha^{*} d \alpha\right\} .
$$

Different from the conventional geometric phase which removes the dynamical phase, this total path includes both the geometric phase and the nonzero dynamical phase with the dynamical phase proportional to the geometric one [16]. As a result, this total phase $\Theta$ is an unconventional geometric phase [16].

When the path is closed, the total displacement operator is rewritten as

$$
D_{t}=D(0) e^{i \Theta}
$$

where the total phase $\Theta$ is only determined by the area of the loop in the phase space, rather than other factors, such as the quantized state of the bosonic mode [17].

According to the definition of the unconventional geometric phase, in the infinitesimal interval $[t, t+d t]$, the 
system governed by the effective Hamiltonian Eq. (5) for different states of the charge qubit evolves as follows,

$$
\left\{\begin{aligned}
|g\rangle\left|\theta_{g}(t)\right\rangle & \rightarrow e^{-i H_{e f f} d t}|g\rangle\left|\theta_{g}(t)\right\rangle=D\left(d \alpha_{g}\right)|g\rangle\left|\theta_{g}(t)\right\rangle, \\
|e\rangle\left|\theta_{e}(t)\right\rangle & \rightarrow D\left(d \alpha_{e}\right)|e\rangle\left|\theta_{e}(t)\right\rangle,
\end{aligned}\right.
$$

where $d \alpha_{g}=i \lambda^{*} e^{-i \delta t} d t, d \alpha_{e}=-i \lambda^{*} e^{-i \delta t} d t$, and $\left|\theta_{u}(t)\right\rangle$ $(u=g, e)$ is the state of the nanomechanical phonon mode, which depends on the qubit state $|u\rangle$ at the time $t$.

If the NAMR is initially in the vacuum state $|0\rangle$, and the charge qubit is prepared in its ground (excited) state $|g\rangle(|e\rangle)$, after an interaction time $t$, the evolution of the system takes the form of

$$
\begin{aligned}
& |g\rangle|0\rangle \rightarrow e^{i \phi_{g}} D\left(\alpha_{g}\right)|g\rangle|0\rangle, \\
& |e\rangle|0\rangle \rightarrow e^{i \phi_{e}} D\left(\alpha_{e}\right)|e\rangle|0\rangle,
\end{aligned}
$$

with

$$
\left\{\begin{array}{l}
\alpha_{g}=i \int_{0}^{t} \lambda^{*} e^{-i \delta t} d t=-\frac{\lambda^{*}}{\delta}\left(e^{-i \delta \tau}-1\right), \\
\alpha_{e}=-i \int_{0}^{t} \lambda^{*} e^{-i \delta t} d t=-\alpha_{g}
\end{array}\right.
$$

and

$$
\left\{\begin{array}{l}
\phi_{g}=\operatorname{Im}\left(\int \alpha_{g}^{*} d \alpha_{g}\right)=-\frac{|\lambda|^{2}}{\delta}\left(t-\frac{\sin (\delta t)}{\delta}\right) \\
\phi_{e}=\operatorname{Im}\left(\int \alpha_{e}^{*} d \alpha_{e}\right)=\phi_{g}
\end{array}\right.
$$

We define the operations in Eq. (12) as unconventional geometric phase gate. Eq. (13) shows that, under the condition of $t=2 l \pi / \delta(l=1,2,3, \cdots)$, the displacement parameter $\alpha_{u}$ for the state $|u\rangle$ moves along a closed path and returns to the original point in the phase space of the coherent state $\left|\alpha_{u}\right\rangle$. Within a definite period of time, e.g. from $t=2 l \pi / \delta$ to $t=2(l+1) \pi / \delta$, the state regarding the phonon mode of the NAMR first evolves from a vacuum state to a coherence state, and then evolves back to the vacuum state again. The generated unconventional geometric phase can be controlled by adjusting the detuning $\delta$ [see Eq. (5)].

We would like to point out that the above geometric phases related to the excited and ground states are the same [see Eq. (14)]. This is trivial since it is a global phase. To get the non-trivial unconventional geometric phase, one can introduce a third level as an auxiliary [18], which is not governed by Hamiltonian (5) and can be used for quantum computation by means of this non-trivial unconventional geometric phase [16, 18, 19, 23, 26, 27].

In our case here, however, we did not induce such a third level and thus the involved unconventional geometric phases are identical for the two levels of the charge qubit. This is because we just focus here on generating entangled cat states between the NAMR and charge qubit by means of the state-dependent displacement operation in a geometric fashion, rather than based on the phases. Nevertheless, the unconventional geometric phase gate operation guarantees the robustness of the entangled cat state for the NAMR and charge qubit in the next section. That results from the fact that the parameter fluctuations can be absorbed into the global phase factor by the unconventional geometric phase gate operations.

\section{THE CAT STATE WITH THE STATE-DEPENDENT DISPLACEMENT OPERATOR}

We show specifically how to generate the entangled cat states of the NAMR by the effective Hamiltonian (5) using the state-dependent displacement operator by the unconventional geometric phase gate described in last section.

Suppose that the initial state of the NAMR is in the vacuum state $|0\rangle$, and the charge qubit is initially in the superposition state $(|g\rangle+|e\rangle) / \sqrt{2}$. The evolution of the system governed by Eq. (12) is given by

$$
\begin{aligned}
\frac{|g\rangle+|e\rangle}{\sqrt{2}}|0\rangle & \rightarrow \frac{e^{i \phi_{g}} D\left(\alpha_{g}\right)|g\rangle+e^{i \phi_{e}} D\left(\alpha_{e}\right)|e\rangle}{\sqrt{2}}|0\rangle \\
& =\frac{e^{i \phi_{g}}|g\rangle\left|\alpha_{g}\right\rangle+e^{i \phi_{e}}|e\rangle\left|\alpha_{e}\right\rangle}{\sqrt{2}} \\
& =\frac{e^{i \phi_{g}}}{\sqrt{2}}\left(|g\rangle\left|\alpha_{g}\right\rangle+|e\rangle\left|-\alpha_{g}\right\rangle\right),
\end{aligned}
$$

which is the entangled state between the NAMR and the charge qubit. In this case, a large enough displacement parameter $\alpha_{u}$ (e.g., $\left|\alpha_{u}\right| \gg 1$ ) for the state $|u\rangle$ guarantees the generated coherent state with a large phonon number, while the geometric phase produced only works as a global phase. Nevertheless, the unconventional geometric phase gate makes sure the robustness in the generation of the entanglement regarding the NAMR cat state.

According to Eqs. (13) and (14), the above entangled state (15) at $t=\pi / 2 \delta$ is reduced to

$$
\frac{|g\rangle+|e\rangle}{\sqrt{2}}|0\rangle \rightarrow \frac{|g\rangle|\alpha\rangle+|e\rangle|-\alpha\rangle}{\sqrt{2}}
$$

where $\alpha=\frac{2 \lambda^{*}}{\delta}=\frac{\Omega g}{2 \delta}\left(\frac{1}{\Delta}+\frac{1}{\Delta+\delta}\right)$ and the global phase has been ignored. The definition of the parameter $\alpha$ shows that the average phonon number in the coherent state can be changed if we adjust the detuning $\delta$. Therefore, it is possible to get very large phonon coherent states in the entangled state which takes a parameter $\alpha \simeq \frac{\Omega g}{\delta \Delta}$ with $\delta \ll|\lambda|$ and $\delta \ll \Delta$ in the ideal condition.

In addition, once we turn off the bias voltage after finishing the operations above, the NAMR and the charge qubit are decoupled. By a single qubit operation, i.e., $|e\rangle \rightarrow(|g\rangle-|e\rangle) / \sqrt{2}$ and $|g\rangle \rightarrow(|g\rangle+|e\rangle) / \sqrt{2}[33]$, on the charge qubit, Eq. (16) changes as

$$
\frac{|g\rangle|\alpha\rangle+|e\rangle|-\alpha\rangle}{\sqrt{2}} \rightarrow \frac{|g\rangle(|\alpha\rangle+|-\alpha\rangle)+|e\rangle(|\alpha\rangle-|-\alpha\rangle)}{2} .
$$


After the measurement is performed on $|g\rangle$ and $|e\rangle$ of the charge qubit, the final states in Eq. (17) collapse into the cat states $(|\alpha\rangle+|-\alpha\rangle) / \sqrt{2}$ and $(|\alpha\rangle-|-\alpha\rangle) / \sqrt{2}$ of the NAMR, respectively, which can be detected by applying a static magnetic field and an alternating current [28].

\section{SIMULATION AND DISCUSSION}

We estimate numerically how well our scheme works under noisy environment. For convenience of the discussion, we take the entangled cat state in Eq. (16) as an example by considering decays from the charge qubit and the NAMR. So the master equation for our scheme is given by

$$
\begin{aligned}
\dot{\rho}= & -i\left[H_{I}, \rho\right]+\frac{\Gamma}{2}\left(2 \sigma \rho \sigma^{+}-\sigma^{+} \sigma \rho-\rho \sigma^{+} \sigma\right) \\
& +\frac{\gamma}{2}\left(2 a \rho a^{\dagger}-a^{\dagger} a \rho-\rho a^{\dagger} a\right),
\end{aligned}
$$

where $\rho$ is the reduced density operator of the system, $\Gamma$ and $\gamma$ are the charge qubit decay rate and the NAMR decay rate, respectively. Note that the thermal phonons of the NAMR have been ignored in Eq. (18), due to the assumption that the thermal phonons can be neglected since the high-frequency NAMR can be remained in its ground state at the work temperature $(T=20 \mathrm{mK})$ of the charge qubit [15, 33].

We define the fidelity of the entangled cat state

$$
F=\langle\Psi|\rho(t)| \Psi\rangle,
$$

where $t$ is the time for producing the entangled cat state, and $|\Psi\rangle$ is a target state relevant to the initial state. In our scheme, the initial state and the target state are $(|g\rangle+$ $|e\rangle)|0\rangle / \sqrt{2}$ and $(|g\rangle|\alpha\rangle+|e\rangle|-\alpha\rangle) / \sqrt{2}$, respectively. Using the realistic parameter values from Refs. 15,28, 30, 3336], we show in Figure 3 the numerical results for the fidelities of the generated state and the corresponding average phonon number $\operatorname{Tr}\left(\rho(t) a^{\dagger} a\right)$ versus the decays of the charge qubit and the NAMR with respect to different Rabi frequencies, where we consider $\pm 10 \%$ change of the Rabi frequency by parameter fluctuations.

Our simulation shows the fidelity for the generated entangled state decreases with the increase of the decays. Here, we suppose $\gamma_{0}=(50 \mu \mathrm{s})^{-1}\left(\Gamma_{0}=(100 \mu \mathrm{s})^{-1}\right)$ as the realistic transmon-type charge qubit (NAMR) decay rate [15, 25]. Figure 3 (a) means that the fidelity of the entangled cat state is sensitive to the decays of the charge qubit and the NAMR, and the unconventional geometric phase gate can be robust against the parameter fluctuation rather than the qubit decay. The decay of the charge qubit causes the flip of the charge qubit states and the decay of the NAMR leads to the phonon leakage from the coherent states.

Moreover, according to the discussion in Section 4, the operation time for preparing the entangled cat state strongly depends on the detuning $\delta$ [see Eq. (13)]. Provided fixed Rabi frequencies regarding the driving fields and the NAMR, the phonon coherent state can be very
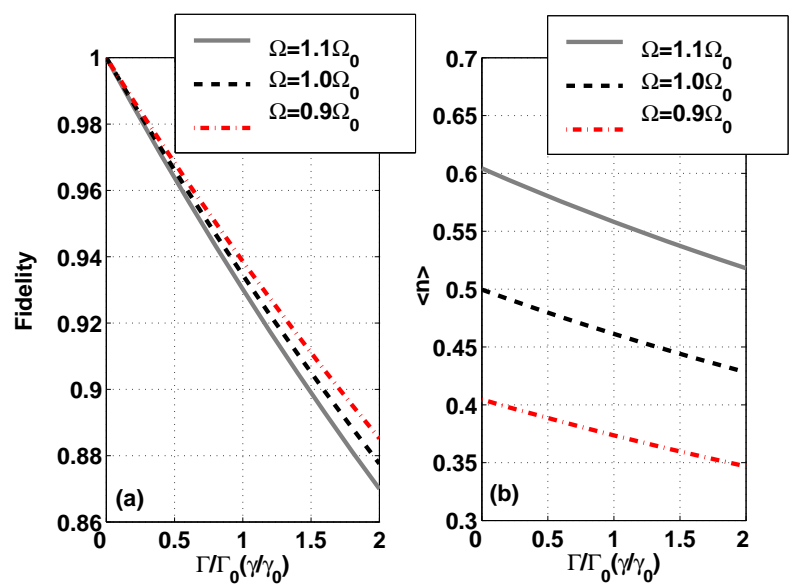

FIG. 3: (Color online) (a) Numerical simulation of the fidelity of the entangled cat state in Eq. (16) versus the NAMR decay and charge qubit decay for different Rabi frequencies. (b) The corresponding average phonon number $\operatorname{Tr}\left(\rho(t) a^{\dagger} a\right)$ in the entangled cat state. $0.9 \Omega_{0}$ and $1.1 \Omega_{0}$ mean $10 \%$ and $-10 \%$ fluctuations, respectively. Here for simplicity we assume $\gamma / \gamma_{0}=\Gamma / \Gamma_{0}$. The parameters take values $\omega_{m}=2 \pi \times 1000 \mathrm{MHz}, Q=10^{5}, g=2 \pi \times 2 \mathrm{MHz}, \Omega_{0}=2 \pi \times 20$ $\mathrm{MHz}, \Gamma_{0}=2 \pi \times 0.02 \mathrm{MHz}, \gamma_{0}=\omega_{m} / Q=2 \pi \times 0.01 \mathrm{MHz}$, $\Delta_{c}=2 \pi \times 200.2 \mathrm{MHz}$, and $\Delta=2 \pi \times 200 \mathrm{MHz}$.

large if $\delta$ is very small. However, we need a long time to achieve the large coherent states in such a case, which is usually longer than the decay time. In contrast, if $\delta$ is very large, the operation time can be very short, but the average phonon number in the entangled state is very small. As a trade-off, the detuning $\delta$ must be chosen carefully to be small enough but make sure the operation to be finished within the decoherence time of the system. Considering the parameters in Figure 3, we may set the operation time $t=1.25 \mu \mathrm{s}$, and then we can find that the lowest average phonon number of the entangled cat state in our simulations is $\langle n\rangle=0.347$ (See the lowest point in red dashed-dotted line in Figure 3 (b)). In the absence of decoherence and parameter fluctuations, the corresponding maximal value of $\langle n\rangle$ in Figure 3 can reach 1.0, which is larger than $\langle n\rangle=0.1$ proposed in Ref. [4].

In addition, there are some other factors affecting the fidelity of the generated entangled cat state under the noisy environment. These factors include the uncertainties and fluctuations of the parameters, which can change the average phonon number in the coherent state. As an example, we have calculated in Figure 3(a) the influence due to $10 \%$ fluctuation in the Rabi frequency, which demonstrates the robustness to the fluctuations to some extent. Particularly, we find that $-10 \%$ fluctuation of the Rabi frequency (i.e., $0.9 \Omega_{0}$ ) corresponds to a higher fidelity of the entangled cat state, which is due to the fact that the average phonon number in the coherent states is proportional to the Rabi frequency $\langle n\rangle=|\alpha|^{2} \sim\left|\frac{\Omega g}{\delta \Delta}\right|^{2}$, when the operation is performed within the time for finishing a quarter of a Rabi oscillation. This implies that 
the less Rabi frequency leads to less average phonons in the coherent state (see Figure 3 (b)), which is less sensitive to the decay of the NAMR. Nevertheless, the simulations in Figure 3 show that the validity of our scheme is restricted by the charge qubit decay, since the scheme based on the geometric phase is never robust against the qubit decay.

\section{CONCLUSION}

In conclusion, we have shown the possibility to achieve the cat state of the NAMR in a NAMR-charge qubit system. The key point of our scheme is the employment of the state-dependent displacement operator based on the unconventional geometric phase gate, which makes the cat state generated with a geometric feature. As a result, our scheme owns some distinct advantages, such as the robustness against the parameter uncertainties and fluc- tuations, the feasibility of the ground state of the NAMR in the work temperature of the charge qubit, independent control of the charge qubit and the NAMR, and the possibility of producing large cat states. In addition, our scheme can also be extended to the Von Neumann measurement based on geometric feature of the operations [37]. Particularly, we argue that our scheme can be achieved using currently available techniques.

\section{Acknowledgments}

JQZ thank Yue Li, Zhi-Jiao Deng, Zheng-Yuan Xue, Zhangqi Yin and Yue-Yue Chen for helpful discussions. This work was supported by the National Natural Science Foundation of China (Grants Nos. 11174027, 11121403, 11274352 and 11304366) and the China Postdoctoral Science Foundation (Grant Nos. 2013M531771 and 2014T70760).
[1] L. F. Wei, Y. X. Liu, C. P. Sun, and F. Nori, Phys. Rev. Lett. 97, 237201 (2006).

[2] F. Marquardt and S. M. Girvin, Physics 2, 40 (2009).

[3] K. Hornberger, S. Gerlich, P. Haslinger, S. Nimmrichter, and M. Arndt, Rev. Mod. Phys. 84, 157 (2012).

[4] Z.-Q. Yin, T. Li, X. Zhang, and L. M. Duan, Phys. Rev. A 88, 033614 (2013).

[5] A. Bassi, K. Lochan, S. Satin, T. P. Singh, and H. Ulbricht, Rev. Mod. Phys. 85, 471 (2013).

[6] S. Nimmrichter and K. Hornberger, Phys. Rev. Lett. 110, 160403 (2013).

[7] E. P. Wigner, Am. J. Phys. 31, 6 (1963).

[8] E. Joos and H. D. Zeh, Z. Phys. B. 59, 223 (1985).

[9] S. Weinberg, Lectures on Quantum Mechanics, (Cambridge University Press, Cambridge, 2013) p.84.

[10] J. Paavola, M. J. W. Hall, M. G. A. Paris, and S. Maniscalco, Phys. Rev. A 84, 012121 (2011).

[11] M. S. Kim, G. Antesberger, C. T. Bodendorf, and H. Walther, Phys. Rev. A 58, R65 (1998).

[12] A. Ourjoumtsev, H. Jeong, R. Tualle-Brouri, and P. Grangier, Nature(London) 448, 784 (2007).

[13] J. R. Friedman, V. Patel, W. Chen, S. K. Tolpygo, and J. E. Lukens, Nature(London) 406, 43 (2000).

[14] C.H. van der Wal et al., Science 290, 773 (2000).

[15] A. D. O'Connell, M. Hofheinz, M. Ansmann, R. C. Bialczak, M. Lenander, E. Lucero, M. Neeley, D. Sank, H. Wang, M. Weides, J. Wenner, J. M. Martinis, and A. N. Cleland, Nature (London) 464, 697 (2010).

[16] S. L. Zhu and Z. D. Wang, Phys. Rev. Lett. 91, 187902 (2003).

[17] S. B. Zheng, Phys. Rev. A 70, 052320 (2004).

[18] J.-Q. Zhang, Y. F. Yu, and Z. M. Zhang, J. Opt. Soc. Am. B 28, 1959 (2011).

[19] Z. Y. Xue, Quantum Inf. Process 11, 1381 (2012); Z. Y. Xue and Z. D. Wang, Phys. Rev. A 75, 064303 (2007).

[20] K. E. Khosla, M. R. Vanner, W. P. Bowen and G. J. Milburn, New J. Phys. 15043025 (2013).

[21] M. V. Berry, Proc. Roy. Soc. Lond. A 392, 45 (1984).

[22] Z.-L. Xiang, S. Ashhab, J. Q. You, and F. Nori, Rev.
Mod. Phys, 85, 623 (2013).

[23] D. Leibfried, B. Demarco, V. Meyer, M. Rowe, A. BenKish, M. Barrett, J. Britton, J. Hughes, W. M. Itano, B. M. Jelenkovic, C. Langer, T. Rosenband, and D. J. Wineland, Nature 422, 412 (2003).

[24] J. Koch, T. M. Yu, J. Gambetta, A. A. Houck, D. I. Schuster, J. Majer, A. Blais, M. H. Devoret, S. M. Girvin, and R. J. Schoelkopf, Phys. Rev. A 76, 042319 (2007).

[25] C. Rigetti, J. M. Gambetta, S. Poletto, B. L. T. Plourde, J. M. Chow, A. D. Corcoles, J. A. Smolin, S. T. Merkel, J. R. Rozen, G. A. Keefe, M. B. Rothwell, M. B. Ketchen, and M. Steffen, Phys. Rev. B 86, 100506 (2012).

[26] C. Y. Chen, M. Feng, X. L. Zhang, and K. L. Gao, Phys. Rev. A 73, 032344 (2006).

[27] X. L. Feng, C. F. Wu, H. Sun, and C. H. Oh, Phys. Rev. Lett. 103, 200501 (2009).

[28] Y. X. Liu, A. Miranowicz, Y. B. Gao. J. Bajer, C. P. Sun, and F. Nori, Phys. Rev. A 82, 032101 (2010).

[29] P. Zhang, Y. D. Wang, and C. P. Sun, Phys. Rev. Lett. 92, 097204 (2005).

[30] D. I. Schuster, A. A. Houck, J. A. Schreier, A. Wallraff, J. M. Gambetta, A. Blais, L. Frunzio, B. Johnson, M. H. Devoret, S. M. Girvin, and R. J. Schoelkopf, Nature (London) 445, 515 (2007).

[31] D. F. V. James, and J. Jerke, Can. J. Phys. 85, 625 (2007).

[32] R. Guzman, J. C. Retamal, E. Solano, and N. Zagury, Phys. Rev. Lett. 96, 010502 (2006).

[33] Y. A. Pashkin, O. Astafiev, T. Yamamoto, Y. Nakamura, and J. S. Tsai, Quantum Inf. Process 8, 55 (2009).

[34] A. N. Cleland and M. R. Geller, Phys. Rev. Lett. 93, 070501 (2004).

[35] P. Rabl, A. Shnirman, and P. Zoller, Phys. Rev. B 70, 205304 (2004).

[36] I. Martin, A. Shnirman, L. Tian, and P. Zoller, Phys. Rev. B 69, 125339 (2004).

[37] G. Vacanti, R. Fazio, M. S. Kim, G. M. Palma, M. Paternostro, and V. Vedral, Phys. Rev. A 85, 022129 (2012). 